PAPER

\title{
The presence of glutamic acid at positions 71 or 74 in pocket 4 of the HLA-DR $\beta 1$ chain is associated with the clinical course of multiple sclerosis
}

\author{
J M Greer, M P Pender
}

J Neurol Neurosurg Psychiatry 2005;76:656-662. doi: 10.1136/jnnp.2004.042168

See end of article for authors' affiliations

....................

Correspondence to: Dr Judith Greer, Department of Medicine, The University of Queensland, Clinical Sciences Building, Royal Brisbane and Women's Hospital, Herston, Queensland 4029,

Australia; i.greer@ medicine.uq.edu.au

Received 25 March 2004 In revised form 13 July 2004

Accepted 9 August 2004

\begin{abstract}
Background: Primary progressive multiple sclerosis (PP-MS) differs from relapsing-remitting or secondary progressive MS (RR/SP-MS) in ways suggesting differences in the pathogenic pathways. Susceptibility to both PP-MS and RR/SP-MS is linked to carriage of the HLA molecule DRB1*1501. Several serologically defined HLA-DR groups (DR1, DR4, DR6, and DR9) occur less often in RR/SP-MS than in controls. Some or all of the HLA-DR molecules encoded by alleles in these serologically defined groups have a negatively charged glutamic acid at residue 71 or 74 of the $\beta 1$ chain $\left(\beta 1_{71} / \beta 1_{74}\right)$. Residues at these positions are important in the formation of pocket 4 in the antigen binding site of the HLA-DR molecule.

Objectives: To investigate whether the presence of alleles encoding HLA-DR molecules containing glutamic acid at $\beta 1_{71} / \beta 1_{74}$ correlates with the course of MS.

Methods: HLA-DR and HLA-DQ alleles and genotypes were analysed in 121 MS patients (50 with PP-MS) and 109 controls by molecular typing.

Results: Alleles encoding HLA-DR molecules containing a glutamic acid at $\beta 1_{71} / \beta 1_{74}$ occurred less often in patients with RR/SP-MS than in those with PP-MS or controls. In subjects not carrying the DRB ${ }^{*} 1501$ allele, a much higher proportion of PP-MS patients carried alleles encoding HLA-DR molecules containing a glutamic acid at $\beta l_{71} / \beta l_{74}$ than did RR/SP-MS patients or controls.

Conclusions: The amino acid residues involved in determining the shape and charge of pocket 4 of the HLA-DR $\beta 1$ chain could influence the clinical course of MS by determining protection against RR/SP-MS or susceptibility to the development of PP-MS.
\end{abstract}

M ultiple sclerosis (MS) is a chronic inflammatory demyelinating disease of the central nervous system (CNS). Typically, MS has a relapsing-remitting course with clinical attacks followed by some improvement and attack-free intervals. However, after 10-15 years of a relapsing-remitting disease course (RR-MS), more than $50 \%$ of patients are likely to have developed a progressive course, characterised by a slow inexorable decline in neurological function. This form of progressive MS is known as secondary progressive MS (SP-MS). In $10-20 \%$ of cases of MS, a progressive course occurs from the onset of disease. This form of MS is known as primary progressive MS (PPMS). PP-MS differs from RR/SP-MS in the amount of CNS inflammation, ${ }^{1}$ age at onset, ${ }^{2}$ the female to male ratio, ${ }^{3}$ the initial symptoms, ${ }^{4}$ and prognosis with regard to disability. ${ }^{2}$ Factors that determine the particular disease course a patient will follow remain obscure, although an older age at onset correlates with a greater likelihood of PP-MS. ${ }^{5}$ Natural history studies, however, show little difference in the progressive phases of PP-MS and SP-MS. ${ }^{2}{ }^{6}$ This and the recent report of a patient with subclinical disease for at least 10 years before the clinical onset of $\mathrm{PP}-\mathrm{MS}^{7}$ are consistent with the suggestion that PP-MS may differ from RR/SP-MS only in having a subclinical initial stage of disease. ${ }^{6}$

Both PP-MS and RR/SP-MS are associated with an increased occurrence of other autoimmune diseases in affected individuals and their first degree relatives, suggesting that they are both autoimmune disorders. ${ }^{8}$ The pathogenesis of MS is thought to involve the development of autoimmunity against components of CNS myelin. This autoimmunity appears to be driven by major histocompatibility complex (MHC) class II restricted T cells, ${ }^{9}{ }^{10}$ which cannot recognise antigen directly but require that it is presented to them on the surface of antigen presenting cells in association with the MHC class II molecules, which in humans are termed human leucocyte antigen (HLA)-DR, HLA-DQ, or HLA-DP. The HLA molecules are formed from two polypeptide chains, $\alpha$ and $\beta$. Some of these chains are extremely polymorphic, particularly the HLA-DR $\beta 1$ chain and the HLA-DQ $\alpha$ and $\beta$ chains. The amino acid sequences of the $\alpha$ and $\beta$ chains of the HLA molecule determine which antigens can be bound and presented to $T$ cells. Immunogenetic studies in MS have shown associations between a limited number of ancestral haplotypes and susceptibility to $\mathrm{MS}^{11-19}$ In particular, the haplotype $\mathrm{DRBI}^{*} 1501-\mathrm{DQAl}^{*} 0102-\mathrm{DQB1}{ }^{*} 0602$ (that is, the 1501 allele of the gene encoding the HLA-DR $\beta 1$ chain, the 0102 allele encoding the HLA-DQ $\alpha$ chain, and the 0602 allele encoding the HLA-DQ $\beta$ chain) correlates with development of MS in northern Europeans, northern European derived populations, Ashkenazi and non-Ashkenazi Jews, Turkish, Mexican Mestizo, and some Japanese and Sardinian patients. However, carriage of these alleles does not correlate with the disease course in MS, as patients with PP-MS and those with RR/SP-MS carry this haplotype in approximately equal proportions. ${ }^{162021}$

Notwithstanding the lack of correlation between DRB1*1501 haplotype and disease course, the finding that specific immune $\mathrm{T}$ cell and antibody responses against myelin and other CNS antigens differ between PP-MS and RR/SP$\mathrm{MS}^{22-25}$ suggests that there may be differences in the other HLA alleles carried by people with different disease courses. However, while some other HLA alleles have been found to be

Abbreviations: $M S$, multiple sclerosis; PP, primary progressive; RR, relapsing-remitting; SP, secondary progressive 
expressed at increased or decreased proportions in either PPMS or RR/SP-MS compared with controls, no significant differences have consistently been found between PP-MS and RR/SP-MS. Several studies have suggested a link between carriage of DR4 alleles and PP-MS, but others have not. This disparity has largely reflected the relatively small sample size of PP-MS in each study (less than 20 individuals) and the large number of HLA alleles being compared. Recently, however, two studies with larger PP-MS sample sizes have been reported-one from Sweden, which tested 85 PP-MS patients, $727 \mathrm{RR} / \mathrm{SP}-\mathrm{MS}$ patients, and 455 controls, ${ }^{5}$ and one from Ireland, which tested 102 PP-MS patients, 202 RR/SPMS patients and 398 controls. ${ }^{21}$ Interestingly, both studies showed significantly decreased frequencies of DRl, DR4, DR6, and DR9 serologically defined HLA groups in RR/SP-MS patients compared with controls, but no significant differences between PP-MS patients and controls; however, this finding was not specifically mentioned in these papers.

The serologically defined HLA nomenclature (DR1, DR2, DR3, and so on) is often used to report HLA-DR results, as it groups together alleles that show similar reactivity to a panel of tissue typing sera. However, it is now known that each of these sera recognise more than one HLA amino acid sequence. For example, the DR4 serum recognises more than 40 different HLA molecules, each differing slightly in the amino acid sequence of the $\beta 1$ chain. These slight differences in sequence can greatly affect the ability of a particular HLA molecule to bind and present an antigen. Thus the finding of decreased frequencies of DR1, DR4, DR6, and DR9 in RR/SPMS patients compared with controls-but no significant differences between PP-MS patients and controls-is of interest because DR1, DR4, DR6, and DR9, together with DR5, are the only serological groupings that contain alleles encoding HLA-DR molecules having a negatively charged glutamic acid at residue 71 or 74 of the $\beta 1$ chain $\left(\beta 1_{71}\right.$ or $\left.\beta 1_{74}\right)$.

These two residues - together with residues 13,70 , and 78 of the $\beta 1$ chain, and residues 9 and 62 of the $\alpha$ chain-are important in determining the shape and charge of pocket 4 of the antigen-binding groove of the HLA-DR molecule ${ }^{2627}$ (fig 1). However, not all of the alleles in DR1, DR4, or DR6 serological groups encode HLA-DR molecules containing a glutamic acid at $\beta 1_{71}$ or $\beta 1_{74}$. As the HLA data in the two large studies were reported only at the level of serological groups, it is not possible to determine whether the presence of a glutamic acid residue at these positions is correlated with the course of MS. The aim of the current study, therefore, was to test the hypothesis that the frequency of HLA-DRBl alleles encoding HLA-DR molecules containing glutamic acid at $\beta 1_{71}$ or $\beta 1_{74}$ differ in PP-MS and RR/SP-MS. The results show that such alleles occur significantly less often in RR/SP-MS than in PP-MS, and support the hypothesis that, in some cases at least, HLA can influence the clinical course of MS

\section{METHODS}

\section{Subjects}

The 230 individuals used in this study included 50 patients with PP-MS, 71 with RR-MS or SP-MS, and 109 unrelated individuals of white ethnicity as controls. More than $95 \%$ of all individuals were of northern European descent, with the remainder being of southern European descent. The MS patients were from Queensland and northern New South Wales in Australia and were seen at the MS clinic, Royal Brisbane and Women's Hospital. All MS patients met the criteria of Poser et $a l^{30}$ for definite MS. All RR/SP-MS patients also met the criteria of McDonald et $a^{l^{31}}$ for MS. Five PP-MS patients did not meet the latter criteria, because magnetic resonance imaging scans were not available for inspection;

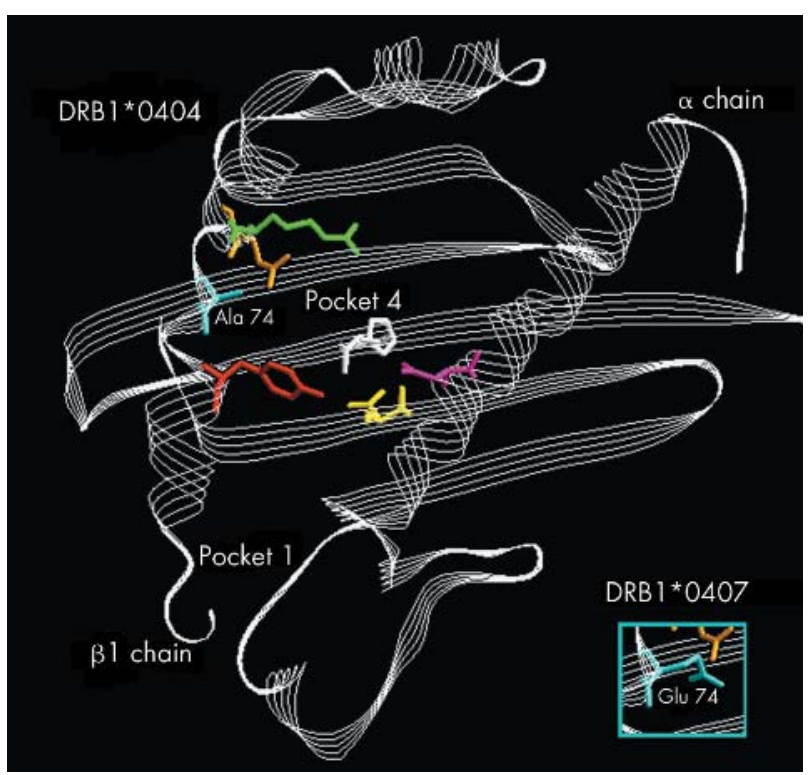

Figure 1 Schematic of the putative antigen binding cleft of the HLADRB 1 ${ }^{*} 0404$ molecule, based on the known crystal structure of

DRB $1{ }^{*} 0401 .{ }^{28}$ One $\alpha$ helix and half of the $\beta$ pleated sheet are formed from the DR $\beta 1$ chain, and the other from the DR $\alpha$ chain. Residues of the peptide antigen that binds in the cleft are usually anchored in pocket 1 , pocket 4 , and pocket 6 or 7 . The residues in pocket 4 that differ from one HLA-DR allele to another are shown in different colours: residues 9 (yellow) and 62 (pink) of the DR $\alpha$ chain and residues 13 (white), 70 (orange), 71 (green), 74 (blue), and 78 (red) of the DR $\beta 1$ chain. HLADRB $1{ }^{*} 0404$ contains an alanine at $\beta 1_{74}$, whereas HLA-DRB $1{ }^{*} 0407$ contains a glutamic acid at this position, as shown in the inset. The figure was prepared using the Swiss PDB viewer. ${ }^{29}$

however, as all these patients had had typical symptoms and signs of PP-MS (with no superimposed relapses) for more than 10 years they were included in the study.

\section{DNA samples}

Peripheral blood samples $(5-10 \mathrm{ml})$ were collected by venepuncture into heparinised tubes, after informed written consent had been obtained from each subject. Red cells were lysed and the cell pellet incubated at $37^{\circ} \mathrm{C}$ overnight in the presence of $0.5 \mathrm{mg} / \mathrm{ml}$ proteinase $\mathrm{K}$. High molecular weight DNA was then salted out, using the method of Miller et al. ${ }^{32}$

\section{HLA typing}

HLA-DRB1, -DQAl, and -DQBl typing was carried out for each DNA sample using Dynal ${ }^{\circledR}$ sequence specific primer sets according to the manufacturer's instructions. Results of the typing are given as specific alleles, and are also grouped according to serologically defined groups listed by the WHO nomenclature committee for factors of the HLA system. ${ }^{33}$

\section{Statistical analysis}

The frequencies of HLA alleles and genotypes in patients and controls were compared using $\chi^{2}$ analysis by $2 \times 2$ contingency tables. Yates's correction was applied when the number of positive samples in any test was less than 5. Fisher's exact test was used when the total number of samples was less than 50. Odds ratios (OR) and 95\% confidence intervals (CI) of the odds ratio for phenotype or genotype frequencies were also calculated. Probability (p) values were corrected for multiple comparisons using Bonferroni's method, according to the formula $\mathrm{p}_{\text {corrected }}\left(\mathrm{p}_{c}\right)=1-(\mathrm{l}-\mathrm{p})^{\mathrm{n}}$, where $\mathrm{p}$ is the uncorrected $\mathrm{p}$ value and $\mathrm{n}$ is the number of comparisons. 


\section{RESULTS}

\section{HLA-DRB 1 phenotype frequencies}

The phenotype frequencies of HLA-DRBl alleles were determined for 50 PP-MS, 71 RR/SP-MS, and 109 non-MS controls. In all, there were 39 different HLA-DRBI alleles in the MS patients or controls in this study. In order to determine if these populations were similar to the two previous large HLA-DR studies, where results were given as serologically defined groups, ${ }^{51}$ the data were analysed and compared with results from those two studies (table 1). In general, the frequencies of serologically defined HLA groups found in our study were within $1-3 \%$ of those found previously. The frequencies that we found for DR6 carriage by all individuals differ more; however, they lie within the range of frequencies found in the two large studies $(25.9 \%$, $22.7 \%$, and $34.9 \%$ for PP-MS, RR/SP-MS, and controls, respectively, in the study from Sweden ${ }^{5}$; and $14.7 \%, 9.4 \%$, and $18.3 \%$ in the study from Northern Ireland ${ }^{21}$ ), and may reflect the mixed northern European ancestry of the white Australian population. In addition, the proportion of RR/SPMS patients carrying DR2 in the current study was larger than in either of the other two studies, although the proportion carrying the DR15 subtype (66\%) again lay between the percentages reported from Sweden $(61 \%)$ and Northern Ireland (69\%). The frequency of carriage of DR2 did not differ in non-MS controls or PP-MS in our study and the other two studies.

There were no significant differences between carriage of different serologically defined HLA groups in PP-MS $v$ RR/SPMS after correction for multiple comparisons. However, as mentioned in the introduction, an interesting observation was that the difference was much more significant (after p values had been corrected for 19 comparisons) between the total number of RR/SP-MS patients (pooled from the present study and the two previous studies) and total number of controls than between the total number of PP-MS patients and the total number of controls for carriage of DRI $\left(\mathrm{p}_{c}=1.6 \times 10^{-3} \quad v \quad \mathrm{p}_{c}=0.999\right), \quad \mathrm{DR} 4 \quad\left(\mathrm{p}_{c}=5.0 \times 10^{-5} \quad v\right.$ $\left.\mathrm{p}_{c}=0.958\right), \quad$ DR6 $\quad\left(\mathrm{p}_{c}=4.2 \times 10^{-3} \quad v \quad \mathrm{p}_{c}=0.767\right), \quad$ and DR9 $\left(\mathrm{p}_{c}=6.7 \times 10^{-3} v \mathrm{p}_{c}=0.999\right)$. This is not just because of the larger numbers of RR/SP-MS patients, and is interesting because a common feature of these serologically defined groups is that they all include alleles that encode HLA-DR molecules containing a glutamic acid at $\beta 1_{71}$ or $\beta 1_{74}$.

None of the other serologically defined HLA-DR groups except DR5 and DR53 (DRB4-that is, a $\beta 4$ chain instead of $\beta 1$ in the HLA molecule, which is present in most DR4, DR7, and DR9 containing haplotypes) includes alleles that encode HLA molecules with glutamic acid residues at residues 71 or 74 of the $\beta 1$ chain. We therefore looked at carriage of alleles that did or did not encode HLA-DR molecules containing a glutamic acid at residues 71 or 74 of the $\beta$ chain by patients or controls in our study (table 2). Whereas $40 \%$ of PP-MS patients and $34.9 \%$ of controls carried alleles encoding HLADRBl molecules that have a glutamic acid at these positions, only $12.7 \%$ of RR/SP-MS patients carried such alleles. Most of this effect is caused by carriage of the DR6 alleles DRBI* 1302 and ${ }^{*} 1401$, which differed significantly $(p=0.01)$ between PP-MS and RR/SP-MS. The results suggest either that carriage of alleles encoding HLA-DR molecules containing a glutamic acid at $\beta 1_{71}$ or $\beta 1_{74}$ is protective against RR/SP-MS, but not against PP-MS, or alternatively that such alleles encode HLA-DRBl molecules that are able to present antigens that are important in the development of PP-MS, but not RR/SP-MS. There were no significant differences between MS patients or controls for carriage of DR53 (table 2).

Within the DR1, DR4, DR5, DR6, and DR9 groups, there were no significant differences among PP-MS patients, RR/ SP-MS patients, or controls for carriage of alleles encoding HLA molecules that do not contain a glutamic acid at $\beta 1_{71}$ or $\beta 1_{74}$. Indeed, all of the other alleles within these groups that were carried by the individuals studied (both MS and controls) encode proteins that have a positively charged arginine or lysine at residue 71 and an alanine at residue 74 .

Most other studies reported using molecular typing for HLA either do not show data for all alleles, or use a mixture of PP-MS and RR/SP-MS patients. However, Zipp et $a l^{34}$ reported full HLA-DR typing for a group of 66 patients with RR/SP-MS and 210 controls. In that dataset, there was also a significant decrease in the proportion of RR/SP-MS patients carrying alleles that encode HLA-DR molecules containing a glutamic acid at $\beta 1_{71}$ or $\beta 1_{74}$ (16 of 66) compared with controls (93 of 210) $\left(\mathrm{OR}=0.4(0.21\right.$ to 0.75$\left.) ; \mathrm{p}_{\mathrm{c}}=0.039\right)$, confirming our observations.

\section{HLA-DRB 1 genotype analysis}

Whereas a small proportion of MS patients are homozygous for their HLA antigens, most express two different alleles at each locus. There is some restriction in the number of different HLA-DRBl genotypes carried by MS patients compared with controls. This is influenced by the increased frequency of the DRB1*1501 allele in MS; in our study, both RR/SP-MS and PP-MS HLA-DR genotypes contained this allele significantly more often $(71.4 \%$ and $62.0 \%$, respectively) than control genotypes (23.9\%; OR for PP-MS $v$ control $=5.2(2.5$ to 10.7$) ; \mathrm{p}_{c}=1.4 \times 10^{-4}$, and OR for RR/SPMS $v$ controls $=8.0(3.9$ to 16.5$) ; \mathrm{p}_{c}=1.5 \times 10^{-7} ; \mathrm{p}$ values are corrected for 42 comparisons). Of the DRB1 genotypes found in PP-MS, 19 did not occur in RR/SP-MS patients. Notably,

Table 1 Phenotype frequencies for HLA-DR in primary progressive multiple sclerosis, relapsing-remitting/secondary progressive multiple sclerosis, and controls

\begin{tabular}{|c|c|c|c|c|c|c|c|c|c|}
\hline \multirow[b]{2}{*}{$\begin{array}{l}\text { Serological } \\
\text { group }\end{array}$} & \multicolumn{3}{|l|}{ PP-MS } & \multicolumn{3}{|l|}{ RR/SP-MS } & \multicolumn{3}{|l|}{ Controls } \\
\hline & $\begin{array}{l}\text { Refs } 5 \text { and } 21 \\
(n=187)\end{array}$ & $\begin{array}{l}\text { Current } \\
(n=50)\end{array}$ & $\begin{array}{l}\text { Total } \\
(n=237)\end{array}$ & $\begin{array}{l}\text { Refs } 5 \text { and } 21 \\
(n=929)\end{array}$ & $\begin{array}{l}\text { Current } \\
(n=71)\end{array}$ & $\begin{array}{l}\text { Total } \\
(n=1000)\end{array}$ & $\begin{array}{l}\text { Refs } 5 \text { and } 21 \\
(n=853)\end{array}$ & $\begin{array}{l}\text { Current } \\
(n=109)\end{array}$ & $\begin{array}{l}\text { Total } \\
(n=962)\end{array}$ \\
\hline 01 & $38(20.3 \%)$ & $10(20 \%)$ & $48(20.3 \%)$ & 137 (14.7\%) & $9(12.7 \%)$ & $146(14.6 \%)$ & $181(21.2 \%)$ & $25(22.9 \%)$ & $206(21.4 \%)$ \\
\hline 02 & $116(62.0 \%)$ & 31 (62\%) & $150(63.3 \%)$ & $591(63.6 \%)$ & $50(70.4 \%)$ & $641(64.1 \%)$ & $277(32.5 \%)$ & $34(31.2 \%)$ & $311(32.3 \%)$ \\
\hline 03 & $49(26.2 \%)$ & $14(28 \%)$ & $63(26.6 \%)$ & $218(23.5 \%)$ & $21(29.6 \%)$ & $239(23.9 \%)$ & $221(25.9 \%)$ & $29(26.6 \%)$ & $250(26.0 \%)$ \\
\hline 04 & $59(31.6 \%)$ & $14(28 \%)$ & $73(30.8 \%)$ & $244(26.3 \%)$ & $18(25.4 \%)$ & $262(26.2 \%)$ & 305 (35.7\%) & $41(37.6 \%)$ & $346(36.0 \%)$ \\
\hline 05 & $11(5.9 \%)$ & $4(8 \%)$ & $15(6.3 \%)$ & 106 (11.4\%) & $8(11.3 \%)$ & $114(11.4 \%)$ & $120(14.1 \%)$ & $24(22.0 \%)$ & $144(15.0 \%)$ \\
\hline 06 & 37 (19.8\%) & $12(24 \%)$ & $49(20.7 \%)$ & $184(19.8 \%)$ & $11(15.5 \%)$ & 195 (19.5\%) & $232(27.2 \%)$ & 23 (21.1\%) & $255(26.5 \%)$ \\
\hline 07 & $20(10.7 \%)$ & $8(16 \%)$ & $28(11.8 \%)$ & $95(10.2 \%)$ & 11 (15.5\%) & $106(10.6 \%)$ & $167(19.6 \%)$ & $19(17.4 \%)$ & $186(19.3 \%)$ \\
\hline 08 & $9(4.8 \%)$ & $2(4 \%)$ & $11(4.6 \%)$ & 79 (8.5\%) & $5(7.0 \%)$ & $84(8.4 \%)$ & $57(6.7 \%)$ & $6(5.5 \%)$ & $63(6.5 \%)$ \\
\hline 09 & $4(2.1 \%)$ & $1(2 \%)$ & $5(2.1 \%)$ & $7(0.8 \%)$ & $0(0 \%)$ & $7(0.7 \%)$ & $24(2.8 \%)$ & $3(2.8 \%)$ & $27(2.8 \%)$ \\
\hline 10 & $4(2.1 \%)$ & $0(0 \%)$ & $4(1.7 \%)$ & $11(1.2 \%)$ & $0(0 \%)$ & 11 (1.1\%) & $12(1.4 \%)$ & $3(2.8 \%)$ & 15 (1.6\%) \\
\hline
\end{tabular}

Pooled data from two previous studies that have tested well defined PP-MS patients ${ }^{521}$ are compared with data from the current study.

MS, multiple sclerosis; PP, primary progressive; RR, relapsing-remitting; SP, secondary progressive. 


\begin{tabular}{|c|c|c|c|c|}
\hline Serological group* & DRB1 allele† & $\begin{array}{l}\text { PP-MS } \\
(n=50)\end{array}$ & $\begin{array}{l}\text { RR/SP-MS } \\
(\mathrm{n}=71)\end{array}$ & $\begin{array}{l}\text { Controls } \\
\text { ( } n=109)\end{array}$ \\
\hline DR1 & 0103 & $2(4 \%)$ & $2(2.8 \%)$ & $4(3.7 \%)$ \\
\hline \multirow{4}{*}{ DR4 } & 0402 & $2(4 \%)$ & $1(1.4 \%)$ & $0(0 \%)$ \\
\hline & 0403 & $0(0 \%)$ & $0(0 \%)$ & $7(6.4 \%)$ \\
\hline & 0406 & $0(0 \%)$ & $0(0 \%)$ & $1(0.9 \%)$ \\
\hline & 0407 & $4(8 \%)$ & $0(0 \%)$ & $6(5.5 \%)$ \\
\hline DR5 & 1103 & $0(0 \%)$ & $0(0 \%)$ & $1(0.9 \%)$ \\
\hline \multirow[t]{6}{*}{ DR6 } & 1301 & $2(4 \%)$ & $3(4.2 \%)$ & $3(2.8 \%)$ \\
\hline & 1302 & $5(10 \%)$ & $2(2.8 \%)$ & $8(7.3 \%)$ \\
\hline & 1401 & $4(8 \%)$ & 1 (1.4\%) & $7(6.4 \%)$ \\
\hline & 1404 & $0(0 \%)$ & $0(0 \%)$ & $1(0.9 \%)$ \\
\hline & 1405 & $0(0 \%)$ & $0(0 \%)$ & $1(0.9 \%)$ \\
\hline & 1407 & $0(0 \%)$ & $0(0 \%)$ & $1(0.9 \%)$ \\
\hline DR9 & 0901 & $1(2 \%)$ & $0(0 \%)$ & $3(2.8 \%)$ \\
\hline \multirow{2}{*}{\multicolumn{2}{|c|}{ Glutamic acid at $\beta 71$ or $\beta 74$ of DRB1 }} & $20(40 \%)$ & $9(12.7 \%) \ddagger$ & $38(34.9 \%)$ \\
\hline & DRB4 (all alleles) & $22(44 \%)$ & $26(36.6 \%)$ & 59 (54.1\%) \\
\hline \multicolumn{5}{|c|}{ 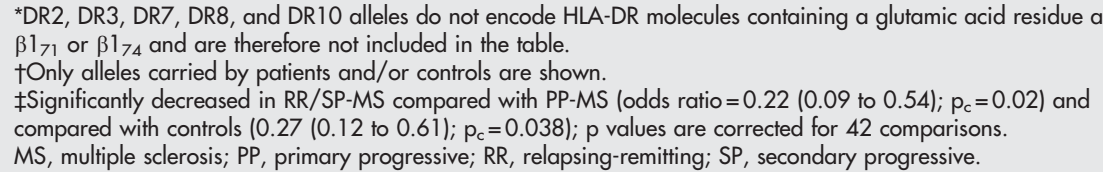 } \\
\hline
\end{tabular}

14 of these 19 genotypes contained an allele encoding HLADR molecules using a glutamic acid at $\beta 1_{71}$ or $\beta 1_{74}$. In contrast, of 15 genotypes occurring in patients with RR/SPMS, but not in patients with PP-MS, only four contained an allele encoding HLA-DR molecules with a glutamic acid at $\beta 1_{71}$ or $\beta 1_{74}$.

The percentages of $\mathrm{DRBI}^{*} 1501$ positive or negative individuals whose genotypes contained an allele encoding a HLA-DRBl molecule with a glutamic acid residue at $\beta 1_{71}$ or $\beta 1_{74}$ are shown in table 3. The percentages of PP-MS patients carrying such alleles were significantly increased compared with the corresponding percentages of RR/SP-MS patients in both the $\mathrm{DRBI}^{*} 1501$ positive and $\mathrm{DRBI}^{*} 1501$ negative groups. In the $\mathrm{DRBI}^{*} 1501$ positive group, this difference was almost entirely caused by DR6 alleles, which made up $78 \%$ of alleles encoding HLA-DR molecules with a glutamic acid residue at $\beta 1_{71}$ or $\beta 1_{74}$; however, in the $\mathrm{DRBl}^{*} 1501$ negative group, both DR4 and DR6 alleles contributed equally. None of the MS patients had genotypes encoding two HLA-DR molecules that both had glutamic acid residues at $\beta 1_{71}$ or $\beta 1_{74}$, but $5 \%$ of controls carried such genotypes.

\section{HLA-DQA 1 and HLA-DQB 1 allele genotype frequencies in PP-MS and RR/SP-MS}

In insulin dependent diabetes mellitus, susceptibility to disease has been linked to carriage of particular HLA-DQ alleles $^{35}$ rather than to the HLA-DR alleles with which they are in linkage disequilibrium. In MS, it has been suggested that HLA-DQ is more closely associated with disease in AfroBrazilian patients than is HLA-DR ${ }^{36}$; however, in white MS patients, HLA-DR linkages appear to be the strongest. We investigated whether there were differences between PP-MS and RR/SP-MS in carriage of HLA-DQ alleles. The frequencies of individuals carrying different HLA-DQAl and HLA-DQB 1 alleles are shown in table 4 . As expected, DQAl*0102 and DQ6 (which includes the allele DQBI*0602)—which are in strong linkage disequilibrium with $\mathrm{DRB1}^{*} 1501$-were found significantly more often in PP-MS $\left(\mathrm{p}_{c}<0.03\right)$ and RR/SP-MS $\left(\mathrm{p}_{c}<5.6 \times 10^{-4}\right)$ than in controls ( $\mathrm{p}$ values corrected for 40 comparisons). There were no significant differences between PP-MS and RR/SP-MS when results were corrected for multiple comparisons. To test further for associations between MS and alleles other than those carried in the HLA-DRB1*1501-DQA1*0102-DQB1*0602 haplotype, allelic frequencies were compared in patients and controls after removal of the DQA1*0102 and DQBI*0602 alleles occurring as part of this haplotype. In the absence of these alleles there were no significant differences in frequency between MS patients and controls or between PP-MS patients and RR/SPMS patients for any HLA-DQ alleles (data not shown).

\section{DISCUSSION}

The question of whether or not the HLA molecules carried by a patient with MS can influence the clinical course of disease

Table 3 Percentages of genotypes that contain alleles encoding HLA-DRB 1 molecules having a glutamic acid residue at position 71 or 74 of the $\beta$ chain

\begin{tabular}{llcll}
\hline Genotype & Group & Number & $\begin{array}{l}\text { Number encoding } \\
\text { Glu71/74 (\%) }\end{array}$ & OR (PP-MS v RR/SP-MS) \\
\hline All & PP-MS & 50 & $20(40 \%)$ & OR=4.6 (1.9 to 11.3) \\
& RR/SP-MS & 71 & $9(12.7 \%)$ & \\
DRB ${ }^{*} 1501$ & Controls & 109 & $43(39.4 \%)$ & OR=3.7 (1.0 to 13.7) \\
positive & PP-MS & 31 & $8(25.8 \%)$ & \\
DRB 1*1501 & RR/SP-MS & 47 & $6(8.5 \%)$ & OR=6.5 (1.7 to 25.3) \\
negative & Controls & 26 & $12(63.2 \%)$ & \\
& RP-MS & 19 & $5(20.8 \%)$ & \\
\hline
\end{tabular}

$M S$, multiple sclerosis; $\mathrm{OR}$, odds ratio; $\mathrm{PP}$, primary progressive; $\mathrm{RR}$, relapsing-remitting; $\mathrm{SP}$, secondary progressive. 
Table 4 Phenotype frequencies for HLA-DQA1 and DQB1 alleles

\begin{tabular}{llll}
\hline $\begin{array}{l}\text { DQA1 } \\
\text { allele }\end{array}$ & $\begin{array}{l}\text { PP-MS } \\
(\mathbf{n}=50)\end{array}$ & $\begin{array}{l}\text { RR/SP-MS } \\
\text { (n=71) }\end{array}$ & $\begin{array}{l}\text { Controls } \\
\text { (n= }=109)\end{array}$ \\
\hline 0101 & $12(24 \%)$ & $9(12.7 \%)$ & $29(26.6 \%)$ \\
0102 & $33(66 \%)^{*}$ & $49(69.0 \%)^{* *}$ & $42(38.5 \%)$ \\
0103 & $3(6 \%)$ & $5(7.0 \%)$ & $5(4.6 \%)$ \\
0104 & $2(4 \%)$ & $0(0 \%)$ & $0(0 \%)$ \\
0105 & $0(0 \%)$ & $1(1.4 \%)$ & $0(0 \%)$ \\
0201 & $7(14 \%)$ & $11(15.5 \%)$ & $23(21.1 \%)$ \\
0301 & $10(20 \%)$ & $17(23.9 \%)$ & $35(32.1 \%)$ \\
0302 & $1(2 \%)$ & $1(1.4 \%)$ & $1(0.9 \%)$ \\
0303 & $4(8 \%)$ & $0(0 \%)$ & $0(0 \%)$ \\
0401 & $1(2 \%)$ & $3(4.2 \%)$ & $5(4.6 \%)$ \\
0501 & $19(38 \%)$ & $29(40.8 \%)$ & $51(46.8 \%)$ \\
0503 & $1(2 \%)$ & $0(0 \%)$ & $0(0 \%)$ \\
0601 & $0 \%$ & $1(1.4 \%)$ & $0(0 \%)$ \\
& & & \\
DQB serological group & & $37(33.9 \%)$ \\
DQ5(1) & $16(32 \%)$ & $13(18.3 \%)$ & $43(39.4 \%)$ \\
DQ6(1) & $38(76 \%)^{* *}$ & $52(73.2 \%)^{* *}$ & $44(40.4 \%)$ \\
DQ2 & $17(34 \%)$ & $28(39.4 \%)$ & $37(33.9 \%)$ \\
DQ7(3) & $12(24 \%)$ & $17(23.9 \%)$ & $6(5.5 \%)$ \\
DQ8(3) & $7(14 \%)$ & $6(8.5 \%)$ & $20(18.3 \%)$ \\
DQ9(3) & $5(10 \%)$ & $7(9.9 \%)$ & $8(7.3 \%)$ \\
DQ4 & $1(2 \%)$ & $4(5.6 \%)$ & \\
\hline & & & \\
\hline
\end{tabular}

${ }^{*} p_{c}<0.03 v$ controls; ${ }^{* *} p_{c}<0.002 v$ controls.

$M S$, multiple sclerosis; PP, primary progressive; RR, relapsing-remitting; $\mathrm{SP}$, secondary progressive.

has been debated now for almost 20 years. Several factors have made it difficult to draw firm conclusions. These have included unclear definition of patient subgroups (such as the grouping of SP-MS and PP-MS), small PP-MS patient numbers, and the analysis of HLA only at the level of serologically defined groups. Most attention has focused on HLA-DRBI*1501, as this allele correlates with susceptibility to disease in numerous studies. ${ }^{11-13} 16202137-39$ However, in HLA heterozygous individuals, who make up the majority of people with MS, the immune system recognises antigen presented not only by $\mathrm{DRBI}^{*} 1501$ but also by the HLA molecules encoded by the other alleles in the genotype. In several other autoimmune diseases-including insulin dependent diabetes mellitus, rheumatoid arthritis, and Addison's disease-it has been shown that combinations of certain alleles in the genotype can affect the development of disease $^{35}{ }^{40}$ or the disease course. ${ }^{41}{ }^{42}$

Our results suggest that the presence of alleles encoding HLA-DR molecules containing a negatively charged glutamic acid residue at $\beta 1_{71}$ or $\beta 1_{74}$ protects against the development of a relapsing-remitting course or increases the susceptibility to a primary progressive course of disease, or both. HLA-DR genotypes consisting of one of these alleles, with or without $\mathrm{DRB1}^{*} 1501$, are significantly decreased in patients with RR/ SP-MS in comparison with those with PP-MS. As the antigens involved in the development of the different clinical forms of MS have not been defined, it is difficult to determine whether these alleles actively participate in the development of a primary progressive course. If, as has been suggested, ${ }^{6}$ PP-MS differs from RR/SP-MS only in that the relapsingremitting phase is subclinical, then another possible interpretation of the data in the current study is that carriage of alleles encoding HLA-DR molecules containing a negatively charged glutamic acid residue at $\beta 1_{71}$ or $\beta l_{74}$ prevents the initial relapsing-remitting phase of disease.

This correlation between the clinical course of MS and the occurrence of alleles encoding HLA-DR molecules containing a negatively charged glutamic acid residue in pocket 4 of the antigen binding groove has not been noted previously because only two studies have been published using a group of more than 20 PP-MS patients, ${ }^{521}$ and for both of these HLA typing was presented only in terms of the serological groups. Thus it is not possible to estimate from those studies the percentage of PP-MS patients carrying alleles containing a negatively charged glutamic acid residue in pocket 4 . There are no other published molecular typing studies using well defined PP-MS patients.

The amino acids found at $\beta 1_{71}$ or $\beta 1_{74}$ of the HLA molecule, together with those at positions 13,70 , and 78 of the $\beta 1$ chain, and at positions 9 and 62 of the $\alpha$ chain, influence the shape and charge of pocket 4 of the particular HLA-DR molecule. ${ }^{26}{ }^{27}$ This is illustrated in fig 1 for $\mathrm{DRBI}^{*} 0404$ and DRBI 0407 , which differ in pocket 4 only at $\beta 1_{71}$; this residue is an alanine (non-charged, hydrophobic) in DRB1*0404 and a glutamic acid (acidic side chain) in $\mathrm{DRBI}^{*} 0407$.

Pocket 4 has been shown to be critically important in the binding of the immunodominant peptide (residues 84-102) of myelin basic protein, a putative autoantigen in $\mathrm{MS}^{43}$ to DRBI*1501 in patients with RR-MS. ${ }^{44}$ For that particular peptide/HLA combination, a large, predominantly hydrophobic uncharged pocket 4 is required to accommodate the large aromatic side chain of a phenylalanine residue. Studies in other MHC class II restricted autoimmune diseases such as rheumatoid arthritis, pemphigus vulgaris, systemic lupus erythematosus, and Hashimoto's thyroiditis have also shown that $\beta 1_{71}$ and/or $\beta 1_{74}$ play a critical role in the recognition of the HLA-DR/peptide complex by $\mathrm{CD}^{+}$helper T cells. ${ }^{45-49}$ In rheumatoid arthritis, for example, the peptides that bind to proteins encoded by $\mathrm{DRBI}^{*} 04$ alleles that are positively $\left(\mathrm{DRB1}{ }^{*} 0404\right)$ or negatively $\left(\mathrm{DRBI}^{*} 0402\right)$ associated with disease have been characterised. ${ }^{49}$ These peptides all have identical requirements at the anchor residues at peptide positions pl and p6, but substantially different requirements at position $\mathrm{p} 4$ (the residue that binds in pocket 4 of the HLA molecule). DRBI*0402, which has a negatively charged glutamic acid at $\beta 1_{71}$, binds peptides with a positive charge at $\mathrm{p} 4$, whereas the presence of the positively charged arginine residue at $\beta 1_{71}$ in $\mathrm{DRBl}^{*} 0404$ correlates with binding of peptides with a negative charge at $\mathrm{p} 4$.

The pocket 4 region of HLA-DR molecules is also important in non-autoimmune inflammatory diseases mediated by $\mathrm{CD}^{+}$helper T cells. For example, the presence of a positively charged arginine at positions $\beta 1_{70}$ or $\beta 1_{71}$ is associated with susceptibility to tuberculoid leprosy. ${ }^{50}$ In contrast, chronic beryllium disease-a lung disease caused by the accumulation in the lung of beryllium specific $\mathrm{CD}^{+}{ }^{+} \mathrm{MHC}$ class II restricted $\mathrm{T}$ cells-is associated with the presence of a glutamic acid at residue 69 of the DPBl molecule. ${ }^{51}$ Residue 69 of the DP $\beta$ chain corresponds to $\beta 1_{71}$ of the DR chain.

Some studies have suggested a trend towards increased carriage of DR4 in patients with PP-MS compared with those with RR/SP-MS, ${ }^{162138}$ although this difference has not reached statistical significance in any study. In the current study, there were no differences in the overall frequency of DR4 alleles in PP-MS compared with RR/SP-MS, although more PP-MS patients carried DRBI*0402 or *0407-which encode HLA-DR molecules containing a glutamic acid residue at $\beta 1_{71}$ or $\beta 1_{74}$-than did RR/SP-MS patients ( $12.0 \% v 1.4 \%$, respectively). In particular, $\mathrm{DRBI}^{*} 1501$ negative PP-MS patients showed an increased frequency of these alleles in the genotype compared with DRB1*1501 negative RR/SP-MS patients $(p=0.036$ by Fisher's exact test $)$. It is therefore possible that the previously reported DR4 association with PP-MS ${ }^{16} 2138$ may have reflected the presence of alleles encoding HLA-DR molecules containing glutamic acid at $\beta 1_{71}$ or $\beta 1_{74}$.

The most obvious effect of the change in shape, size, or charge of pocket 4 would be on the ability of the particular HLA molecule to bind a disease relevant peptide; however, it 
is also possible that the DR4, DR6, and other HLA molecules that contain a glutamic acid at $\beta 1_{71}$ or $\beta 1_{74}$ are encoded by alleles that are in linkage disequilibrium with some other site within the MHC region which could affect the disease course. We did not find any evidence to suggest that HLA-DQ alleles account for the differences seen between PP-MS and RR/SPMS (table 4); however, the HLA region is also in strong linkage disequilibrium with genes encoding, for example, the cytokine tumour necrosis factor (TNF) and the molecule $\mathrm{I} \kappa \mathrm{BL}$, a member of the I $\mathrm{KB}$ family of inhibitory factors that are involved in control of the pro-inflammatory transcription factor, NF- $\kappa B$. Several polymorphisms are known to occur in TNF and IKBL genes. ${ }^{52}$ Patients carrying HLA alleles in linkage disequilibrium with such polymorphisms could have altered inflammatory immune responses, thus leading to a different clinical course of disease.

Not all PP-MS patients carried alleles encoding HLA-DR molecules containing a glutamic acid residue at $\beta 1_{71}$ or $\beta 1_{74}$, and some RR/SP patients did carry such alleles. This probably reflects the heterogeneity in disease pathogenesis. Overall, however, there was a clear cut difference between the two groups of MS patients, which suggests that-in some cases at least-the HLA genotype can exert an influence on the clinical course of MS. The results from the study of Zipp et $a^{34}$ and from the large serotyping studies from Sweden ${ }^{5}$ and Northern Ireland ${ }^{21}$ provide indirect support for this hypothesis.

\section{ACKNOWLEDGEMENTS}

We would like to thank Lynn Mallard, Diane Muller, and Peter Csurhes for excellent technical assistance. This work was supported by grants from the National Multiple Sclerosis Society (RG 3190-A-1), and MS Australia.

\section{Authors' affiliations}

J M Greer, M P Pender, Neuroimmunology Research Centre, School of Medicine, The University of Queensland, Herston, Queensland, Australia

M P Pender, Department of Neurology, Royal Brisbane and Women's Hospital, Herston

Competing interests: none declared

\section{REFERENCES}

1 Thompson AJ, Kermode AG, MacManus, et al. Patterns of disease activity in multiple sclerosis: clinical and magnetic resonance imaging study. BMJ 1990;300:631-4.

2 Cottrell DA, Kremenchutzky M, Rice GPA, et al. The natural history of multiple sclerosis: a geographically based study. 5 . The clinical features and natural history of primary progressive multiple sclerosis. Brain 1999;122:625-39.

3 Bashir K, Whitaker JN. Clinical and laboratory features of primary progressive and secondary progressive MS. Neurology 1999;53:765-71.

4 McDonnell GV, Hawkins SA. Primary progressive multiple sclerosis: increasing clarity but many unanswered questions. J Neurol Sci 2002; 199:1-15.

5 Masterman T, Ligers A, Olsson T, et al. HLA-DR15 is associated with lower age at onset in multiple sclerosis. Ann Neurol 2000;48:21 1-19.

6 Ebers GC. Natural history of primary progressive multiple sclerosis. Mult Scler 2004; 10(suppl 1):S8-15.

7 McDonnell GV, Cabrera-Gomez J, Calne DB, et al. Clinical presentation of primary progressive multiple sclerosis 10 years after the incidental finding of typical magnetic resonance imaging brain lesions: the subclinical stage of primary progressive multiple sclerosis may last 10 years. Mult Scler 2003;9:204-9.

8 Henderson RD, Bain CJ, Pender MP. The occurrence of autoimmune diseases in patients with multiple sclerosis and their families. J Clin Neurosci 2000;7:434-7

9 Martin R, McFarland HF, McFarlin DE. Immunological aspects of demyelinating diseases. Annu Rev Immunol 1992;10:153-87.

10 Hellings N, Raus J, Stinissen P. Insights into the immunopathogenesis of multiple sclerosis. Immunol Res 2002;25:27-51.

11 Stewart GJ, Basten A, Guinan J, et al. HLA-Dw2, viral immunity and family studies in multiple sclerosis. J Neurol Sci 1977;32:153-67.

12 Hauser SL, Fleischnick E, Weiner HL, et al. Extended major histocompatibility complex haplotypes in patients with multiple sclerosis. Neurology 1989;39:275-7.
13 Hillert J, Olerup O. Multiple sclerosis is associated with genes within or close to the HLA-DR-DQ subregion on a normal DR15, DQ6, Dw2 haplotype. Neurology 1993;43:163-8.

14 Saruhan-Direskeneli G, Esin S, Baykan-Kurt B, et al. HLA-DR and -DQ associations with multiple sclerosis in Turkey. Hum Immunol 1997:55:59-65.

15 Marrosu MG, Murru R, Murru MR, et al. DRB1-DQA1-DQB1 loci and multiple sclerosis predisposition in the Sardinian population. Hum Mol Genet 1998;7:1235-7.

16 Weinshenker BG, Santrach P, Bissonet AS, et al. Major histocompatibility complex class II alleles and the course and outcome of MS. A populationbased study. Neurology 1998;51:742-7.

17 Kwon OJ, Karni A, Israel S, et al. HLA class II susceptibility to multiple sclerosis among Ashkenazi and non-Ashkenazi Jews. Arch Neurol 1999;56:555-60.

18 Karni A, Kohn Y, Safirman C, et al. Evidence for the genetic role of human leukocyte antigens in low frequency DRB ${ }^{*} 1501$ multiple sclerosis patients in Israel. Mult Scler 1999;5:410-15.

19 Alvarado-de la Barrera C, Zuniga-Ramos J, Ruiz-Morales JA, et al. HLA class II genotypes in Mexican Mestizos with familial and nonfamilial multiple sclerosis. Neurology 2000;55:1897-900.

20 Olerup O, Hillert J, Fredrikson S, et al. Primarily chronic progressive and relapsing/remitting multiple sclerosis: two immunogenetically distinct disease entities. Proc Natl Acad Sci USA 1989;86:7113-17.

21 McDonnell GV, Mawhinney H, Graham CA, et al. A study of the HLA-DR region in clinical subgroups of multiple sclerosis and its influence on prognosis. J Neurol Sci 1999;165:77-83.

22 Greer JM, Csurhes PA, Cameron KD, et al. Increased immunoreactivity to two overlapping peptides of myelin proteolipid protein in multiple sclerosis. Brain 1997; 120:1447-60.

23 Sadatipour BT, Greer JM, Pender MP. Increased circulating antiganglioside antibodies in primary and secondary progressive multiple sclerosis. Ann Neurol 1998;44:980-3.

24 Pender MP, Csurhes PA, Wolfe NP, et al. Increased circulating T cell reactivity to $G M 3$ and GQ1b gangliosides in primary progressive multiple sclerosis. J Clin Neurosci 2003;10:63-6.

25 Lefranc D, Almeras L, Dubucquoi S, et al. Distortion of the self-reactive lgG antibody repertoire in multiple sclerosis as a new diagnostic tool. J Immunol 2004; 172:669-78

26 Stern LJ, Brown JH, Jardetzky TS, et al. Crystal structure of the human class II MHC protein HLA-DRI complexed with an influenza virus peptide. Nature 1994;368:215-21.

27 Fu XT, Bono CP, Woulfe SL, et al. Pocket 4 of the HLA-DR $\left(\alpha, \beta 1{ }^{*} 0401\right)$ molecule is a major determinant of $\mathrm{T}$ cell recognition of peptide. J Exp Med $1995 ; 181: 914-26$

28 Hennecke J, Wiley DC. Structure of a complex of the human $\alpha / \beta$ T cell receptor (TCR) HA1. 7, influenza hemagglutinin peptide, and major histocompatibility complex class II molecule, HLA-DR4 (DRA*0101 and $\left.\mathrm{DRB} 1{ }^{*} 0401\right)$ : Insight into TCR cross-restriction and alloreactivity, J Exp Med 2002;195:571-81.

29 Guex N, Peitsch MC. SWISS-MODEL and the Swiss-Pdb Viewer: an environment for comparative protein modeling. Electrophoresis 1997; 18:2714-23.

30 Poser CM, Paty DW, Scheinberg L, et al. New diagnostic criteria for multiple sclerosis: guidelines for research protocols. Ann Neurol 1983;12:227-31.

31 McDonald WI, Compston A, Edan G, et al. Recommended diagnostic criteria for multiple sclerosis: guidelines from the International Panel on the Diagnosis of Multiple Sclerosis. Ann Neurol 2001;50:121-7.

32 Miller SA, Dykes DD, Polesky HF. A simple salting out procedure for extracting DNA from human nucleated cells. Nucleic Acids Res 1988;16:1215-18.

33 Schreuder GMT, Hurley CK, Marsh SGE, et al. The HLA Dictionary 2001: a summary of $\mathrm{HLA}-\mathrm{A},-\mathrm{B},-\mathrm{C},-\mathrm{DRB} 1 / 3 / 4 / 5,-\mathrm{DQB} 1$ alleles and their association with serologically defined HLA-A, $-B,-C,-D R$ and $-D Q$ antigens. Tissue Antigens 2001;58:109-40.

34 Zipp F, Windemuth C, Pankow $\mathrm{H}$, et al. Multiple sclerosis associated amino acids of polymorphic regions relevant for the HLA antigen binding are confined to HLA-DR2. Hum Immunol 2000;61:1021-30.

35 Stenström G, Berger B, Borg H, et al. HLA-DQ genotypes in classic type 1 diabetes and in latent autoimmune diabetes of the adult. Am J Epidemiol 2002;156:787-96

36 Caballero A, Alves-Leon S, Papais-Alvarenga R, et al. DQB $1 * 0602$ confers genetic susceptibility to multiple sclerosis in Afro-Brazilians. Tissue Antigens 1999;54:524-6.

37 Olerup O, Hillert J. HLA class II-associated genetic susceptibility for multiple sclerosis: a critical evaluation. Tissue Antigens 1991;38:1-15.

38 Hillert J, Grönning M, Nyland H, et al. An immunogenetic heterogeneity in multiple sclerosis. J Neurol Neurosurg Psychiatry 1992;55:887-90.

39 Celius EG, Harbo HF, Egeland T, et al. Sex and age at diagnosis are correlated with the HLA-DR2,DQ6 haplotype in multiple sclerosis. J Neurol Sci 2000;178:132-5.

40 Myhre AG, Undlien DE, Løvås K, et al. Autoimmune adrenocortical failure in Norway: autoantibodies and human leukocyte antigen class II associations related to clinical features. J Clin Endocrinol Metab 2002;87:618-23.

41 Knip M, Kukko M, Kulmala $P$, et al. Humoral beta-cell autoimmunity in relation to HLA-defined disease susceptibility in preclinical and clinical type 1 diabetes. Am J Med Genet 2002;115:48-54

42 Wassmuth R, Wagner U. Prognostic use of human leukocyte antigen genotyping for rheumatoid arthritis susceptibility, disease course, and clinical stratification. Rheum Dis Clin North Am 2002;28:17-37.

43 Bielekova B, Goodwin B, Richert N, et al. Encephalitogenic potential of the myelin basic protein peptide (amino acids 83-99) in multiple sclerosis: results of a phase II clinical trial with an altered peptide ligand. Nat Med 2000;6: 1167-75. 
44 Smith KJ, Pyrdol J, Gauthier L, et al. Crystal structure of HLA-DR2 (DRA*0101, DRB $1^{*} 1501$ ) complexed with a peptide from human myelin basic protein. $J$ Exp Med 1998;188:1511-20.

45 Djoulah S, Busson M, Sasazuki T, et al. A new predictive model for insulindependent diabetes mellitus susceptibility based on combinations of molecula HLA-DRB1 and HLA-DQB1 pockets. Tissue Antigens 1999:54:341-8.

46 Haas J-P, Nevinny-Stickel C, Schoenwald U, et al. Susceptible and protective major histocompatibility complex class II alleles in early onset pauciarticular juvenile chronic arthritis. Hum Immunol 1994;41:225-33.

47 Wucherpfennig KW, Yu B, Bhol K, et al. Structural basis for major histocompatibility complex (MHC)-linked susceptibility to autoimmunity: charged residues of a single MHC binding pocket confer selective presentation of selfpeptides in pemphigus vulgaris. Proc Natl Acad Sci USA 1995;92:11935-9.
48 Wang FW, Yu ZQ, XY JJ, et al. HLA and hypertrophic Hashimoto's thyroiditis in Shanghai Chinese. Tissue Antigens 1988;32:235-6.

49 Hammer J, Gallazzi F, Bono E, et al. Peptide binding specificity of HLA-DR4 molecules: Correlation with rheumatoid arthritis association. J Exp Med 1995; 181:1847-55

50 Zerva L, Cizman B, Mehra NK, et al. Arginine at positions 13 or 70-71 in pocket 4 of HLA-DRB1 alleles is associated with susceptibility to tuberculoid leprosy. J Exp Med 1996;183:829-36.

51 Richeldi L, Sorrentino R, Saltini C. HLA-DPB1 glutamate 69: a genetic marker of Beryllium disease. Science 1993:262:242-4.

52 Price $\mathrm{P}$, Witt $\mathrm{C}$, Allcock R, et al. The genetic basis for the association of the 8.1 ancestral haplotype (A1, B8, DR3) with multiple immunopathological diseases. Immunol Rev 1999; 167:257-74.

\section{NEUROLOGICAL STAMP}

\section{Robert Bárány 1876-1936}

$\mathrm{R}$ obert Bárány was educated at the University of Vienna, graduating in medicine in 1900. In 1903, he accepted an appointment at the University of Vienna otology clinic working in the department of Adam Politzer, who had established the speciality in Austria. Here he began his studies exploring the equilibrium system of the ear and the brain. Among his contributions was a test, now called the Bárány test, for diagnosing diseases of the semicircular canals of the inner ear by syringing the ear with either hot or cold water. He noted nystagmus when the water was above or below, but not at body temperature. He concluded that with caloric nystagmus, the endolymph, normally at body temperature, displaced itself when it came into contact with water at other temperatures, and that the vestibular nerve transmitted the thermal disturbance within the endolymph to the brain. His work led to the development of the Bárány rotating chair, which demonstrated the relationship between the vestibular system, the nervous system, and centres within the cerebellum. In 1913, he investigated the somatopic localisation in the cerebellum and postulated that the vermis was concerned with coordinated movements of the trunk, the hemisphere with the extremities, and the flocculus with movements of the eyes.

Bárány received the Nobel Prize in 1914 for his development of clinical investigation of the human equilibrium system. He essentially founded a new area of medical science and practice. At the time of this award, which was delayed until 1915 by the outbreak of World War I, he was a prisoner of war in Siberia. When Austria entered the war, Bárány saw an opportunity to investigate his ideas with brain injured soldiers. He volunteered for medical service and was assigned to the fortress of Przemysl in Galicia (now Poland) to provide care for 123000 men. There he built an otolaryngology unit, and developed a very successful primary suture technique for the suture of head wounds. He was captured there by advancing Russian troops in April 1915 and taken prisoner. After Prince Carl of Sweden persuaded Czar Nicholas 1 to release Bárány, the Swedish Red Cross negotiated his release and emigration to Sweden. He became director of the otorhinolaryngology clinic at the University of Uppsala, Sweden in 1917, and in 1926 became a full professor there. In 1921 he became the first to describe positional vertigo.

Upon his death, the University of Uppsala established the Bárány medal, to be awarded at 5 year intervals to the most

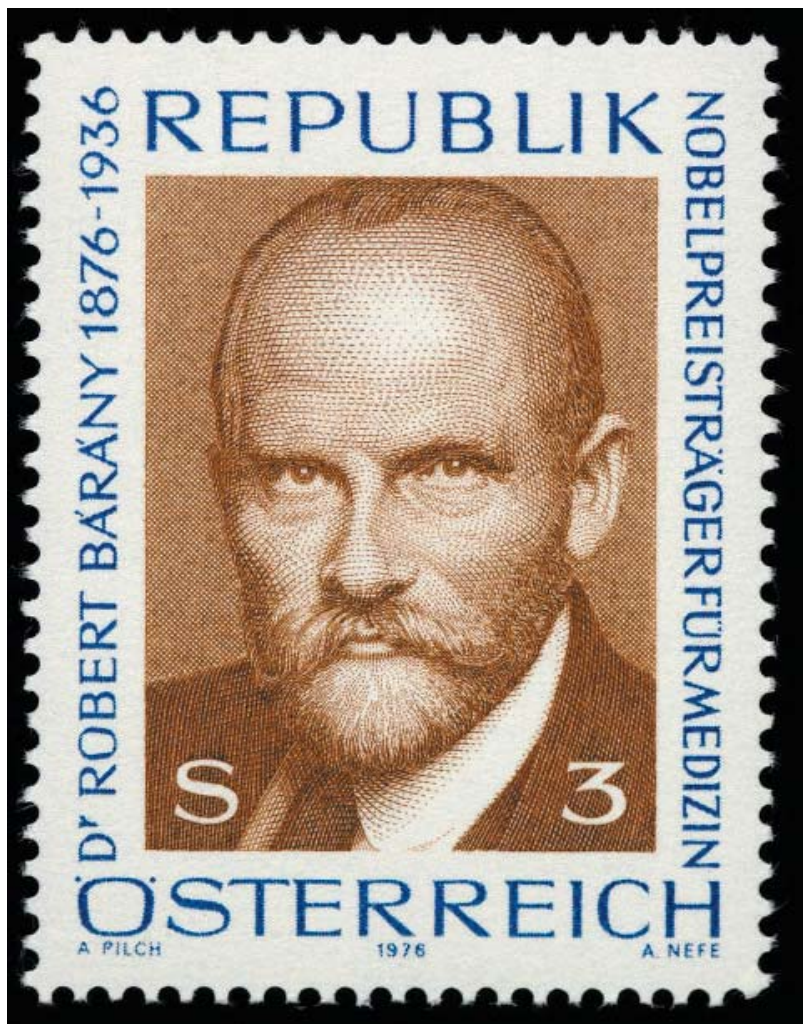

outstanding scientist in the field of the vestibular research. The Bárány society was founded in his honour by $\mathrm{C} \mathrm{S}$ Hallpike and C O Nylen in 1960.

Bárány is portrayed here on a stamp issued by Austria in 1976, the centenary of his birth (Stanley Gibbons no. 1756, Scott no. 1031).

L F Haas

Department of Neurology, Wellington Hospital, Private Bag 7902, Riddiford Street, Wellington, New Zealand; Ihaas@xtra.co.nz 